\title{
A master KLASS in laparoscopic gastrectomy
}

$\mathrm{D}$ espite the rapid progress in the molecular understanding of gastric cancer and the development of targeted therapies to treat it, currently, the only treatment option to improve the survival rate of patients with this malignancy is surgical resection. Nonetheless, a limited use of gastrectomy can be enough to treat these patients successfully. Unfortunately, because of a lack of large-scale studies reporting the long-term outcomes for patients with gastric cancer who are treated with laparoscopic gastrectomy, this approach is still questioned by many surgeons. Now, the Korean Laparoscopic Gastrointestinal Surgical Society (KLASS) group, led by Sang-Uk Han, has demonstrated that curative laparoscopic gastrectomy leads to the same long-term outcomes in patients with all stages of gastric cancer as noted with open surgery.

"Since the first case of laparoscopyassisted distal gastrectomy was reported in 1994, it has been used widely to treat gastric cancer due to the well-known short-term benefits, such as low rates of morbidities, decreased pain, shorter length of hospital stay, and, less estimated blood loss," says Han. After this seminal study in 1994, several studies to assess early stage gastric cancer also showed a superior postoperative recovery in

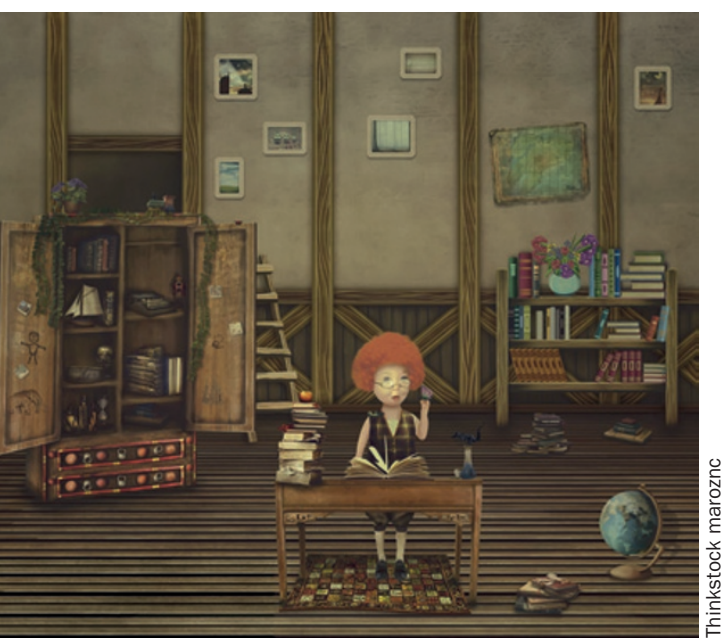

patients treated with laparoscopic surgery compared with those treated with open surgery. But because these studies were small-sized, single-centred, analyses of the long-term outcomes, multicentred data from a large-scale trial was required before laparoscopy could become a widely applicable surgery for most patients with gastric cancer. To that end, the KLASS group initiated the KLASS-01 prospective study: a multicentre, phase III, prospective randomized trial. However, "before the prospective study, we needed background data that could support and justify this trial. Thus, we reviewed the multicentre data of ten institutions retrospectively and analysed them to evaluate the feasibility and safety of laparoscopic gastrectomy," explains Han.

To prepare such background, 10 surgeons retrospectively collected data from their gastric cancer surgeries. This included data from laparoscopic and open procedures collected from the date of their first laparoscopic surgery for gastric cancer until December 2005. "These 10 participating surgeons were from multiple high-volume centres performing at least 80 gastrectomies per year in Korea," clarifies Han. This approach eliminated institutional and referral-based bias as well as ensuring surgeon expertise. The data collected included clinical, surgical, and long-term follow-up results of all patients who underwent curative gastrectomy for gastric cancer at the participating institutions. The cohort included 2,976 patients-1,477 laparoscopic and 1,499 open gastrectomies carried out over a period of more than 7 years.

"These data were analysed by a medical statistician, who matched all cases for extent of resection, lymphadenectomy, BMI, surgeons, and stage of tumour," explains Han.

The authors found no difference between the two approaches regarding overall survival, disease-specific survival, and recurrence-free survival with the exception of patients with stage IA disease, for whom there was an improved overall survival in the laparoscopic group (95.3\% in the laparoscopic group and $90.3 \%$ in open group; $P<0.001)$. This difference, however, could be attributed to selection bias, despite matching, as the patients who were selected for open procedures tended to be older and had different location of their tumours.

\section{1 ...before the prospective study, we needed background data that could support and justify this trial 17}

The morbidity and mortality of the case-matched group was not statistically different either and the mean learning curve was 42 , which means that 42 patients would need to be treated by a surgeon in 1 year in order to achieve mastery of the technique and improve the lymphadenectomy skills, decrease the number of complications and, overall, improve outcomes.

The retrospective analysis by the KLASS group concluded that laparoscopic gastrectomy is a minimally invasive approach that, while providing a sound long-term outcome, can be mastered in high-volume gastric cancer centers and should be offered to those patients with early, distal-type gastric cancers.

The results from this retrospective study should be confirmed by the prospective randomized controlled trials currently underway by the KLASS group for early-stage gastric cancer (KLASS-01; NCT00452751) and subtotal gastrectomy with D2 lymph-node dissection for locally advanced-stage gastric cancer (KLASS-02; NCT01456598).

\section{Teresa Villanueva}

